\title{
Research on biofilm formation and the treatment of river water by the bionic plants
}

\author{
Qiankun Hong*, Shixia Zhang, Shan Li \\ Zhejiang Tongji Vocational College of Science and Technology, Hangzhou, 311231, China
}

\begin{abstract}
In order to explore the effect of bionic plants constructed with different fillers on the removal of pollutants in river waters, this study made sponge filler and biological rope filler into bionic plants, and compared the biofilm attached to the two bionic plants through long-term field film tests. The bionic plants that were successfully coated with the film were subjected to an indoor purification test to prove the removal of COD, ammonia nitrogen and other pollutants in the water by the bionic plants.
\end{abstract}

\section{Introduction}

The formation and development of cities are closely related to rivers. As an important factor of urban ecological balance, urban river is an important natural geographic element in the urban landscape and one of the important ecological corridors ${ }^{[1]}$. It is also the green lifeline of the city, with water supply, flood control and drainage, water transportation, Tourism and entertainment, providing green space, beautifying the environment, regulating climate, maintaining natural ecology, culture and entertainment, etc., can reduce the urban heat island effect, enrich the diversity of urban landscape and urban species, expand the space for urban development, and create cultural and sports entertainment for citizens. The space close to nature plays an irreplaceable key role ${ }^{[2]}$.

However, with the rapid development of economy, population increase, industrialization and urbanization, the scale of cities is increasing, which leads to the increasing demand for and dependence on water resources of cities, and the accompanying deterioration of water quality is also becoming more and more serious. The color and smell of many river water bodies have deteriorated to varying degrees, and some rivers have even become sewage channels, resulting in water bodies showing reduced dissolved oxygen, increased nutrients, increased water temperature changes, and reduced environmental capacity, and other symptoms of river ecosystem degradation, and water quality The deterioration of the river has caused the basic extinction of fish and shrimps and other organisms in the river, the simplification of the aquatic community structure, the decline of biodiversity, especially the disappearance of some species that are beneficial to humans or of potential value. The increasing degradation of urban river ecosystems, the continuous weakening of ecological functions, and the decline in landscape quality have seriously affected the physical and mental health of residents on both sides of urban rivers ${ }^{[3]}$, and have posed an increasingly serious threat to human survival and social and economic development. Therefore, Regarding the improvement of urban rivers, it has become a major environmental issue of concern to the whole society. At the same time, the treatment of urban river pollution and the restoration of degraded river ecosystems have also become current research in the field of aquatic ecosystems.

In the face of increasingly serious river pollution, my country has carried out various measures including river dredging, diversion dilution, river aeration, biological restoration, etc. to improve the water environment quality of urban rivers, but the current situation is still very severe. Due to the multi-channelization of rivers in many cities in my country, poor water flow, sludge accumulation, and serious degradation of the selfpurification function of the rivers, the above measures still have many shortcomings in practical applications. For example, for urban rivers, due to the slower water flow and higher pollutant concentration, if the physical means such as water diversion and aeration are used for purification, the cost is too high, and the symptoms can not be cured. Therefore, seeking efficient, safe and easyto-use bio-ecological restoration technology suitable for urban river governance has become a prerequisite for river governance. Among them, bio-ecological restoration technology is one of the key technologies for the treatment of river water pollution in the city. It has many advantages such as safety, economy, practicability, and systemicity. It has become the main technical means for river pollution treatment, especially for For heavily polluted "desertification" rivers, bio-ecological restoration technology has become a key technology for the water environment management of many urban rivers $^{[4]}$.

At present, biological-ecological restoration technologies mainly include constructed wetland,

*Corresponding author: hongqk0918@163.com 
oxidation pond, buffer zone, physical ecological engineering, ecological floating bed and other technologies ${ }^{[6]}$. Bio-ecological restoration technology mainly relies on the absorption and assimilation of nutrient elements such as nitrogen and phosphorus by plant roots, stems, leaves and other tissues, as well as the degradation, transformation, and decomposition of microorganisms attached to plant roots to remove pollutants, so as to achieve water purification. Therefore, the good growth of plants and the attachment of microorganisms to plant roots are the core and key of bio-ecological restoration technology. However, the pollution status of urban rivers in my country with low oxygen, low transparency, and high ammonia nitrogen has become a stress factor for the growth of aquatic plants, making aquatic plants grow poorly or even unable to grow, resulting in poor water heterogeneity and lack of habitat and attachment of indigenous microorganisms in the water body The urban watercourse presents a landscape of "desertification", which seriously affects the self-purification ability of the urban watercourse.

Based on the above status, a series of ecological projects including ecological floating beds have been carried out in recent years to achieve enhanced purification of polluted river water quality, and some results have been achieved. However, due to the scarcity of urban land resources in my country, it may be difficult to establish a sufficient area of constructed wetlands and buffer zones at this stage. Although ecological floating beds can achieve in-situ treatment of water pollutants, floating bed plants face root systems. Short growth, secondary pollution caused by plant decay in nongrowing seasons, floating bed management and other issues have become the main bottlenecks that limit the large-scale application of this technology. Therefore, new technical methods are sought to repair the severely degraded The city's river ecosystem is looming.

The emergence of bionic plants has made up for the bottleneck of traditional bio-ecological restoration technology in water pollution control, and provided new technical means for ecological restoration of heavily polluted urban rivers. Bionic plants, also known as "artificial aquatic plants" or "ecological fillers", imitate the form of natural aquatic plants and the main functions of aquatic plants in water purification, and combine the biofilm technology in natural water bodies with the filler technology of traditional sewage treatment. Through various fiber processing to form new water treatment materials, build "underwater forests" in heavily polluted "desertification" rivers, provide suitable habitats for indigenous microorganisms in polluted water bodies, and promote microorganisms to gather, grow, multiply, and metabolize, thereby Degrade pollutants to achieve the purpose of water purification. As a new water ecological restoration technology, bionic plants have unique advantages: (1)They have a huge specific surface area (according to reports, $1 \mathrm{~m} 3$ reservoir plants can provide a surface area of up to $245 \mathrm{~m} 2$ ), and can create a surface area suitable for various organisms. Ecological microenvironment ${ }^{[7]}$; (2)It is not affected by the seasons, and can avoid the turbulent changes in water quality caused by the seasonal alternation of aquatic plants: (3) It is not restricted by the degree of water pollution, which can avoid the low transparency, low dissolved oxygen and pollutants of aquatic plants. Current status of poor growth in high-concentration water bodies; (4)Reusable. Based on the above characteristics, since the advent of bionic plants, they have been rapidly studied and applied in practice. However, bionic plants made of different filler materials have different effects on river treatment. In view of this, this study selects two kinds of fillers commonly used in the market and makes bionic plants. By comparing the field membrane biomass and indoor purification test effects of the two bionic plants, we are looking for better bionic plants, hoping to be bionic plants. It is used to provide theoretical basis for river pollution control.

\section{Materials and methods}

\section{1. materials and production of the bionic plant}

In this study, sponge fillers and biological rope fillers, which are widely used in the market, are used as biomimetic plant raw materials. The two fillers were inserted into the steel wire separately. The length of the filler inserted into each steel wire was $80 \mathrm{~cm}$, and the spacing between the fillers was controlled to $15 \mathrm{~cm}$. The lower end nodes of the steel wire were connected and fixed to the bottom of the river. On the steel wire net, each steel wire net is connected with 25 steel wire sets of test fillers.

\subsection{Field test}

The film hanging cycle is 120 days. After the film is successfully applied, the bionic plants are taken out, and they are $10 \mathrm{~cm}$ and $40 \mathrm{~cm}$ underwater respectively. Sampling at $70 \mathrm{~cm}$ is used to determine biomass. Six bionic plants of the two fillers were randomly selected and put into sterile bags for later indoor purification tests.

\subsection{Indoor purification test}

The test container is a plastic drum with a diameter of 55 $\mathrm{cm}$ and a height of $73 \mathrm{~cm} .6$ bionic plants which with two fillers were selected randomly, and a blank water body without bionic plants was used as a control. The water body used in the indoor test is configured according to the water quality of the River, and the water quality indicators are shown in Table 1. 
Table 1. Indicators of simulated sewage.

\begin{tabular}{|c|c|}
\hline Element & Range \\
\hline $\mathrm{COD} /\left(\mathrm{mg} \cdot \mathrm{L}^{-1}\right)$ & $189-241$ \\
\hline $\mathrm{TN} /\left(\mathrm{mg} \cdot \mathrm{L}^{-1}\right)$ & $20.19-23.01$ \\
\hline $\mathrm{NH}_{4}{ }^{+}-\mathrm{N} /\left(\mathrm{mg} \cdot \mathrm{L}^{-1}\right)$ & $14.26-15.83$ \\
\hline $\mathrm{TP} /\left(\mathrm{mg} \cdot \mathrm{L}^{-1}\right)$ & $1.52-1.67$ \\
\hline
\end{tabular}

\subsection{Analyse method}

The weight of the biofilm attached to bionic plants is measured by filtration, drying, and mass weighing; COD is measured by permanganate index method; ammonia nitrogen is measured by Nessler's reagent method.

\section{Results and discussion}

\section{1 membrane biomass on the bionic plant}

Bionic plants can provide suitable habitats for aquatic microorganisms in rivers and provide carrier conditions for the aggregation and metabolism of microorganisms due to their large specific surface area. As shown in Figure 1, there is a significant difference in the amount of biofilm attached to the two bionic plants. Among them, the biomass on the sponge filler is higher than that on the biological rope filler. This may be due to the higher specific surface area of the sponge filler, which can provide more for microorganisms. Contact opportunities, and more microorganisms grow on the sponge filler. In addition, the biomass on the two bionic plants showed obvious stratification, and both increased with the increase of water depth. This was mainly due to the increase of the microbial biomass in the river water body as the water depth increased, and the adhesion of the bionic plants The biomass also increased.

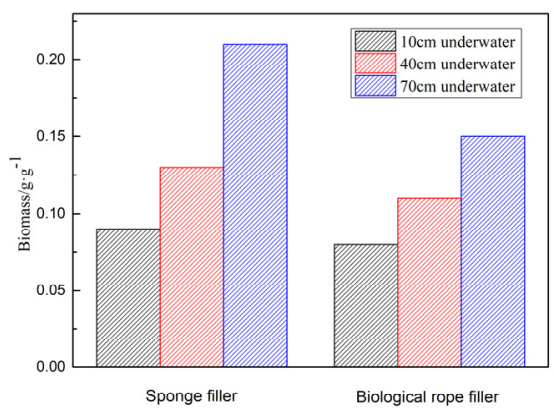

Fig. 1. Biomass attached to bionic plants.

\subsection{Indoor purification test results of bionic plants}

\subsubsection{The removal of $C O D$}

Put the bionic plants that have been successfully filmed in the field into the indoor reactor and run for 15 days. The COD removal effect is shown in Figure 2. It can be seen that the two bionic plants have a good removal effect on COD, mainly due to surface adhesion. The result of the metabolism of the biofilm. However, the removal effect of sponge filler on COD is stronger than that of biological rope filler, which is mainly due to the increase of the biomass of sponge filler in the early stage.

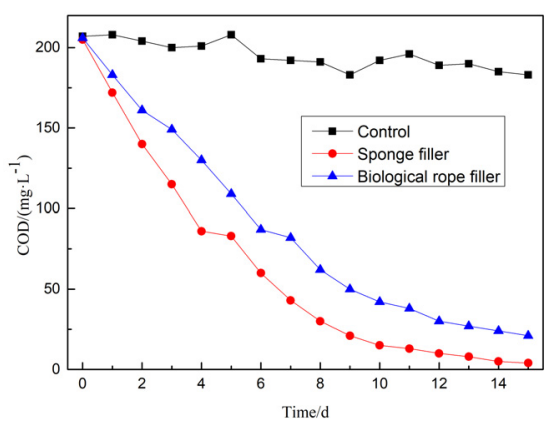

Fig. 2. The removal effect of bionic plants on COD.

\subsubsection{Removal of NH4+-N}

Bionic plants are wastewater in-situ treatment technologies developed in recent years, mainly using biological carriers shaped like aquatic plants to adsorb and multiply indigenous microorganisms in water bodies, create habitats for microorganisms, and magnify microbial biomass by tens or hundreds of times. Makes the carrier-attached microbial cell mass concentration tens to thousands of times higher than that of the suspension system, and enables the microbes to continue to reproduce and renew themselves, which will provide important for the adhesion of the nitrogen cycle functional flora of the heavily polluted river with poor heterogeneity ${ }^{[8]}$. Space for growth, and ultimately promote the continuous degradation of nitrogen in the water body. In addition, bionic plants have unique advantages in the in-situ degradation process of pollutants in heavily polluted rivers: (1) Bionic plants have a huge specific surface area and can create an ecological microenvironment suitable for the survival of various organisms. Attachment offers the possibility. The core of bionic plants for degradation of water pollutants is the attached microbial membrane. In traditional sewage treatment processes, the biofilm method has become a successful technology. Its core is to provide a carrier for the attachment of denitrification functional microorganisms in the wastewater treatment structure, so that the microorganisms can be attached. A large number of growth and formation of biofilm on the carrier filler, strengthen and use the metabolism of microorganisms to remove water pollutants ${ }^{[9]}$. 
The removal effect of bionic plants on ammonia nitrogen in the water body is shown in Figure 3. The two bionic plants have a relatively significant removal effect on ammonia nitrogen in the water body. At the initial stage of removal, the ammonia nitrogen decreased significantly, which may be due to the adsorption and adsorption of biofilms in the bionic plants. The comprehensive results of nitrification, and then gradually tend to be flat, which is mainly based on biodegradation. Similarly, because the amount of biomass attached to the sponge filler is higher, the nitrification effect is stronger than that of the biological rope filler.

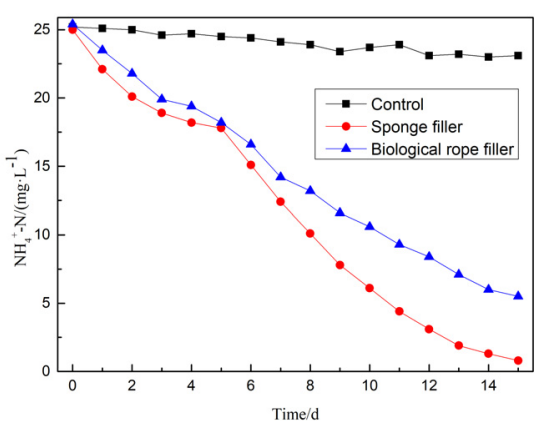

Fig. 3. The removal effect of bionic plants on $\mathrm{NH}_{4}{ }^{+}-\mathrm{N}$

\section{4 conclusion}

(1) Different bionic plants have an important influence on the adhesion of biofilms. The sponge filler used in the experiment has higher specific surface area and higher attached biomass, and the biofilm formed by bionic plants has obvious stratification effect;

(2) The indoor purification test shows that the biomass attached to the bionic plants determines the removal of COD and ammonia nitrogen. The larger the biomass, the higher the removal rate of COD and ammonia nitrogen.

\section{Acknowledgements}

This work was supported by the Science and Technology Project of Zhejiang Water Resources Department (NO. RC1943); the Science and Technology Project of Zhejiang Water Resources Department (NO. RC1830) and "the Fundamental Research Funds for the Colleges" (NO. FRF2019005) .

\section{References}

1. J. X. Song, B. Tang, J. L. Zhang, X. Y. Dou, Q. Liu, W. B. Shen. System dynamics simulation for optimal stream flow regulations under consideration of coordinated development of ecology and socioeconomy in the Weihe River Basin, China. Ecological Engineering, 2018, 124: 51-68.

2. W. J. Tian, F. H. Hao, C. Wang, J. B. Zhai. Study on Dynamic Biofilm Forming Process of Bionic Packing in Streams. China water \& waster water, 2007, 23: 81-83.
3. B. Liu, X. Du, G. X. Wang, L. M. Zhang. Study on treatment of municipal waster by the bionic plants. Environmental Pollution \& Control, 2012, 34: 1619.

4. H. Wu, Z. Jian, P. Li, et al. Nutrient removal in constructed microcosm wetlands for treating polluted river water in northern China. Ecological Engineering, 2011, 37: 560-568.

5. S. Kouki, F. M'Hiri, N. Saidi, et al. Performances of a constructed wetland treating domestic wastewaters during a macrophytes life cycle. Desalination, 2009, 246: 452-467.

6. G. X. Wang, L. M. Zhang, H. Chua, et al. A mosaic community of macrophytes for the ecological remediation of eutrophic shallow lakes. Ecological Engineering, 2009, 35: 582-590.

7. L. Zhu, Z. Li, T. Ketola. Biomass accumulations and nutrient uptake of plants cultivated on artificial floating beds in China's rural area. Ecological Engineering, 2011, 37: 1460-1466.

8. Y. F. Liao, J. Y. You, C. G. Lu. Biomimetic medium purifies rural micro-polluted water sources. Environmental Science and Management, 2009, 34: 95-97.

9. R. Zhang, Z. Y. Han, Z. J. Chen, et al, Microstructure and microbial ecology of biofilm in the bioreactor for nitrogen removing from wastewater: A review. Chinese journal of ecology. 2011, 30: 2628-2636. 